R. Kern · D. Varjú

\title{
Visual position stabilization in the hummingbird hawk moth, Macroglossum stellatarum L. I. Behavioural analysis
}

\begin{abstract}
Optomotor responses of freely flying hawk moths, Macroglossum stellatarum, were characterized while the animals were hovering in front of and feeding on a dummy flower. Compensatory translational and rotational movements of the hawk moth were elicited by vertical grating patterns moving horizontally, mimicking imposed rotational and translational displacements of the animal in the horizontal plane. Oscillatory translational and rotational pattern motion leads to compensatory responses that peak in the frequency range between $2 \mathrm{~Hz}$ and $4 \mathrm{~Hz}$. The control systems mediating the translational and rotational components of the optomotor response do not seem to influence each other. The system mediating translational responses is more sensitive in the fronto-lateral part of the visual field than in the lateral part; the opposite is true for the rotational system. The sensitivity of the translational system does not change along the vertical, whereas the rotational system is much more sensitive to motion in the dorsal than in the ventral part of the visual field. These sensitivity gradients may reflect an adaptation to the specific requirements of position stabilization in front of flowers during feeding.
\end{abstract}

Key words Vision - Optomotor behaviour · Position stabilization · Hawk moth - Insect

Abbreviations $R P M$ rotational pattern motion $\cdot R R$ rotational response $\cdot T P M$ translational pattern motion $\cdot T R$ translational response

\section{R. Kern $(\bowtie)^{1} \cdot$ D. Varjú}

Lehrstuhl für Biokybernetik, Universität Tübingen,

Auf der Morgenstelle 28, D-72076 Tübingen, Germany

\section{Present address:}

${ }^{1}$ Lehrstuhl für Neurobiologie,

Fakultät für Biologie, Universität Bielefeld,

Postfach 1001 31, D-33501 Bielefeld, Germany

Fax: + $49(0) 521 / 106-6038$

e-mail: roland.kern@biologie.uni-bielefeld.de

\section{Introduction}

Flying animals use visual cues to control their flight course (cf. David 1986; Collett et al. 1993; Egelhaaf and Borst 1993; Reichert 1993). Disturbances of the course, as may be elicited by a gust of wind, induce translations and rotations of the animal along and around its body axes. These translations and rotations induce characteristic changes of the spatio-temporal brightness pattern on the retina, i.e. changes of the so-called optic flow. The characteristic optic flow is generally assumed to be evaluated by the nervous system in order to mediate responses compensating for the disturbance.

Compensatory responses mediated by the visual system have been studied in great detail (reviews: Wehner 1981; Collett et al. 1993). Most of the behavioural studies were performed with tethered flying animals, measuring either head movements, abdominal deflections or torque responses of the whole animal. This approach served to unravel important aspects about the computational mechanisms underlying the compensatory optomotor responses (reviews: Heisenberg and Wolf 1984; Egelhaaf and Borst 1993; Hengstenberg 1993; Reichert 1993). However, experiments on tethered flying animals leave undecided the significance of the recorded compensatory responses for flight control under normal conditions. Here, the spatio-temporal brightness changes on the retinae may be much more complex than those the animal is confronted with under laboratory conditions. Therefore, the significance of the compensatory responses can only be assessed by freeflight experiments. Unfortunately, under free-flight conditions it is usually difficult to distinguish between, for example, intended deviations from a straight course and deviations that result from external disturbances. Only when manipulations of the sensory input can be related to the responses of the animals, is it possible to analyse compensatory optomotor behaviour under freeflight conditions. This approach has been employed in only a few studies where large parts of the visual field of 
the animal were moved, thereby simulating the visual consequences of a disturbance of the animal's flight course (Collett and Land 1975; David 1979a,b; Collett 1980a,b; Wagner 1986).

The diurnal hummingbird hawk moth, Macroglossum stellatarum, is able to hover. The reason for hovering is immediately obvious: Macroglossum feed on the wing, sucking nectar from flowers via its long proboscis (Knoll 1922). In its natural environment, the animal visits up to 1500 flowers a day (Pfaff 1991), demonstrating precision and speed when moving from flower to flower at intervals of a few seconds. When feeding, the animal compensates for disturbances of its position relative to the flower, caused, for instance, by a blast of wind drifting the animal and/or the flower (Pfaff 1991). In order to respond adequately to the translational and rotational disturbances, Macroglossum has to extract the necessary information from the spatio-temporal brightness changes on the retina.

How information might be extracted from optic flow has been investigated intensively both theoretically and experimentally. In theoretical studies it has been shown that any flow-field can be decomposed mathematically into a translational and a rotational component (e.g. Koenderink and van Doorn 1976; Longuet-Higgins and Prazdny 1980; Rieger 1983). Being able to perform such a decomposition is advantageous in order to compensate for disturbances most efficiently. In addition, it has been shown that under certain conditions there are distinct areas of the visual field in which the optic flow induced by translation of the animal is only weakly affected by simultaneous rotation and vice versa (cf. Dahmen et al. 1997). Therefore, image motion in the corresponding parts of the retinae can be attributed to translation or rotation of the animal, respectively, and thus compensated for without being much affected by the other optic flow component. For example, the control of angular orientation of cruising hoverflies has been proposed to be governed by motion in those regions of the visual field which coincide with the direction of flight, because in these regions there is no translational component of image motion (Collett 1980a). Several animal species have been demonstrated not to respond equally strongly to image motion in all parts of the visual field (crab: Kunze 1963; Sandeman 1978; Nalbach and Nalbach 1987; Kern et al. 1993; waterstrider: Junger and Dahmen 1991; backswimmer: Blanke 1996; bee: Moore et al. 1981; fly: Blondeau and Heisenberg 1982; locust: Spork and Preiss 1993; moth: Preiss 1991; pigeon: Nalbach 1992). The strength of the optomotor response to simulated translatory or rotatory self-motion rather depends on the stimulated area of the retina. In order to infer from the optic flow whether a disturbance can be compensated for by a rotation or a translation, it might be advantageous to not only focus on a single retinal area but also to combine the motion information from more than one retinal area. Such interactions of signals originating from distant parts of the visual field have been found in several animal species (see Discussion).
In our experiments the visual consequences of disturbances experienced by Macroglossum in natural situations were simulated. Macroglossum readily becomes accustomed to feed on dummy flowers that can be connected to a reservoir of honey solution. The animal keeps feeding for periods of up to a minute, allowing the behavioural response to be recorded for a sufficiently long period for a detailed characterization. We analysed the dynamic properties of the optomotor responses to both translational and rotational disturbances. Another set of experiments addressed the question of whether different eye regions are specialized for mediating translational or rotational responses. Furthermore, it was investigated whether the detection of translation and rotation is based on specific interactions of the motion signals from different parts of the retinae. Since translational and rotational disturbances are likely to be superimposed under natural conditions, we also asked whether the compensation of each component of the composite disturbances is affected by the presence of the other. Finally, the characteristic properties of the optomotor system of Macroglossum are discussed as adaptations to its peculiar feeding behaviour. In a companion paper (Kern 1998), the potential neuronal substrate mediating the optomotor responses characterized in the present study is analysed under similar stimulus conditions.

\section{Materials and methods}

Animals

All experiments were conducted with the European hawk moth, M. stellatarum L. (Lepidoptera, Sphingidae). A detailed description of rearing and keeping the animals can be found elsewhere (Farina et al. 1994; Kern 1994)

\section{Setup}

The experimental setup was placed on a large table within the breeding cage of the animals. The stimulus patterns were displayed on two computer monitors (NEC MultiSync 3FG, frame repetition rate set to $60 \mathrm{~Hz}$ ) subtending an angle of $70^{\circ}$ (Fig. 1). A blue plastic disk (dummy flower) was mounted between the two monitors. It was glued to the tip of a horizontal pipe which was connected to a large reservoir of honey solution. A small hole (diameter about $0.15 \mathrm{~cm}$ ) in the centre of the disk (diameter $0.6 \mathrm{~cm}$ ) allowed the animals to insert their proboscis and to suck honey solution. The vertical position of the dummy flower coincided with the horizontal midlines of the monitor screens. In the horizontal plane, the dummy flower was positioned in such a way that the perpendiculars raised onto the anterior edges of the pattern screens intersected in the middle of the blue plastic disk (dotted lines in Fig. 1).

In order to reduce the number of stationary contours in the visual field of the animals, several measures were taken. A sheet of translucent paper was fixed above the monitors and the dummy flower. It scattered the light of the translucent light tubes (Osram Biolux) illuminating the cage and reduced the contrast of objects at the ceiling. In addition, the table which supported the monitors was covered with black cloth. Large pieces of black cardboard were fixed beside the monitor screens and in the rear of the setup. A $1 \mathrm{~cm}$ wide horizontally aligned strip of white paper was fixed at the height of the dummy flower on the cardboard and the screens. It 


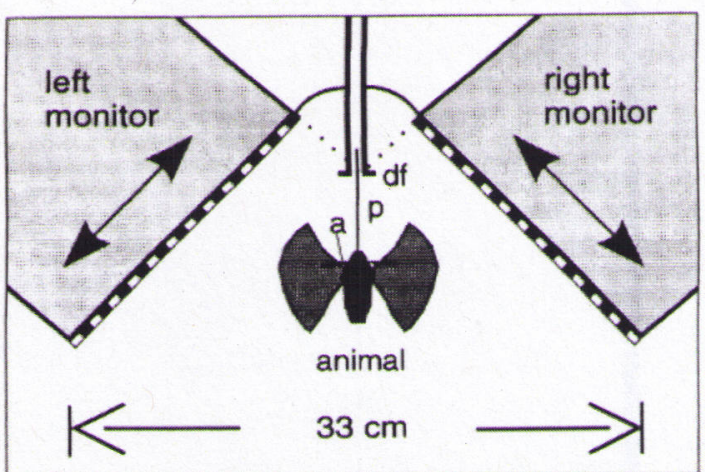

Fig. 1 Schematic drawing of the experimental situation seen from above (not drawn to scale). Two monitors subtended an angle of $70^{\circ}$. Between the monitors a dummy flower $(d f)$ was mounted (diameter $0.6 \mathrm{~cm}$ ). The vertical position of the dummy coincided with the horizontal midlines of the monitor screens. In the horizontal plane the perpendiculars onto the anterior edges of the screens (dotted lines, length $5.7 \mathrm{~cm}$ ) intersected in the middle of the dummy. On the screens square-wave gratings of vertical black and white stripes (indicated by the checkered bars) were generated. The gratings could be moved from front to back or back to front (indicated by the double-arrows). The animal was hovering in front of the dummy flower while sucking honey solution from it. At the side and in the rear of the setup black cardboard was fixed to occlude surrounding stationary contours (not shown). ( $p$ proboscis, $a$ antennae)

has been suggested earlier that Macroglossum needs at least one horizontal contour to perform a stable flight (Farina et al. 1995).

Stimulation

On the screens (size: $21 \mathrm{~cm} \times 14 \mathrm{~cm}$ ) black-and-white square-wave gratings (pattern contrast about $90 \%$; mean luminance about $165 \mathrm{~cd} \mathrm{~m}^{-2}$ ) of variable height and width were generated (program written in TurboPascal, running on two $16-\mathrm{MHz}$ PC AT286). The patterns on both screens could be moved horizontally. By moving both the left and right pattern from front to back or back to front, translational motion of the animal along its long axis was simulated. By moving the patterns from back to front on one screen and from front to back on the other screen, rotational motion was simulated. In some experiments the animals were stimulated unilaterally. In these cases one monitor was switched off or its screen was covered with black cardboard. The patterns were oscillated sinusoidally. The oscillation frequency was $1 \mathrm{~Hz}$ in all experiments except those in which the dynamic properties of the behavioural responses were analysed.

The angular extent of a particular pattern, as seen by the animal, varied with the position and orientation of the animal. Since the stimuli were presented on flat screens the angular size of both the pattern wavelength and the oscillation amplitude, as seen by the animal, was not constant either. For an animal hovering between the screens at a distance of $1 \mathrm{~cm}$ from the dummy, the angular extent of a pattern wavelength of $2 \mathrm{~cm}$ decreased from about $18^{\circ}$ at the anterior edge to about $2^{\circ}$ at the posterior edge of each screen. Corresponding conditions hold for the oscillation amplitude. The maximal value of the angular pattern wavelength and oscillation amplitude as present in the anterior part of the screens was used to characterize the stimulus pattern. In some experiments the grating patterns did not cover the entire monitor screen and consisted of two spatial periods only. In these experiments the patterns were oscillated around different azimuthal positions in order to probe the spatial sensitivity distribution of the optomotor responses. Therefore, the angular extent of the pattern as seen by the animal was kept almost constant in these experiments by adjusting its width (in $\mathrm{cm}$ ) according to its azimuthal position on the monitor screen. The oscillation amplitude was adjusted accordingly.
In order to specify the angular size of the patterns the following coordinate system is used: in azimuth, $0^{\circ}$ is in the direction of the dummy flower, $90^{\circ}$ is lateral to the right. Azimuth values will be given only for the right monitor screen; they are mirror symmetrical for the left monitor. In elevation, $0^{\circ}$ corresponds to the height of the dummy and the horizontal midlines of the screens, positive angles correspond to locations above, negative ones to those below the midlines. The angular size and spatial wavelength of the various stimulus patterns were as follows: 'whole-screen patterns' (wavelength $2 \mathrm{~cm}, 18.4^{\circ}$ ) covered the screens entirely and extended from $49^{\circ}$ to $128^{\circ}$ in azimuth and from $-45^{\circ}$ to $+45^{\circ}$ in elevation. Patterns displayed exclusively in the upper or lower halves of the screens ranged from $49^{\circ}$ to $128^{\circ}$ in azimuth; the vertical extent of the upper pattern ranged from $0^{\circ}$ to $+45^{\circ}$, that of the lower one from $-45^{\circ}$ to $0^{\circ}$ (wavelength $2 \mathrm{~cm}$ ). Patterns with a horizontal width of only two periods had a vertical extent ranging from $+45^{\circ}$ to $-45^{\circ}$. While stationary, these patterns covered an azimuthal area from $61^{\circ}$ to $83^{\circ}$ ('fronto-lateral pattern'; wavelength $1.4 \mathrm{~cm}, 12^{\circ}$ ) or from $86^{\circ}$ to $105^{\circ}$ ('lateral pattern'; wavelength $1.8 \mathrm{~cm}, 11^{\circ}$ ). If in motion, these patterns oscillated around their resting position with an amplitude of half their wavelength $(0.7 \mathrm{~cm}$ and $0.9 \mathrm{~cm}$, respectively).

The motion stimuli were intended to simulate the visual consequences of self-motion of an animal in a stationary surround. However, since the moving patterns were generated on flat screens, the retinal image motion differs from what an animal would experience during real self-motion. When the animal rotates about its vertical axis, the retinal image of the entire panorama moves with the same angular velocity, whereas in the present experiments when simulating rotation of the animal - the angular velocity of the moving patterns is not the same along the horizontal axis of the screens (see above). Thus, the retinal slip induced by the moving patterns cannot be compensated for entirely in all parts of the visual field. Nevertheless, the stimuli employed are sufficient to elicit compensatory optomotor responses.

\section{Recording}

The animals were video-taped (Sony, U-matic Videocassette Recorder VO 5800PS, sampling rate $50 \mathrm{~Hz}$ ) from above (Figs. 1, 2) through a small circular hole in the translucent cover above the monitors. To improve the contrast between the brownish animal and the background, a round piece of white cardboard was placed on the black cloth below the dummy flower.

\section{Acquisition of raw data}

The position and the orientation of the head in the horizontal plane were determined by automatic frame-by-frame analysis (program written in TurboPascal) of the video tapes. Compensatory responses of the animal were decomposed into a translational and a rotational component. The translational component was calculated as the time-dependent change in distance between the snoot of the animal and the dummy flower. The rotational component was determined as the change of the orientation of the head with respect to an external coordinate system ('head angle') over time. In order to increase resolution, the angle of the head was not calculated from two image points only. Rather, it was calculated from the coordinates of the snoot and the average coordinates of a group of image points on the back part of the head, each point being detected by a threshold operation. In principle, the rotational component could have been determined by the change in the orientation of the whole animal rather than by the change in head angle. However, since the abdomen is used for flight control when responding to both translational (acceleration and deceleration) and rotational stimulation (steering), parts of the body and the head were temporarily disaligned, especially when the stimulus patterns were oscillated at higher frequencies. Therefore, the head angle rather than the body axis has been taken as a measure of the rotational response component. 
Processing of raw data

Since the patterns were oscillated sinusoidally, the amplitude of the fundamental frequency component was determined for each response trace: sinusoids with the oscillation frequency of the pattern were fitted to the measured time-dependent distance or head angle. The fit is based on a least-square method and compensates for linear drift (algorithm developed by H. Dahmen; program written in TurboPascal). The method was shown to be reliable by calculating the relative deviation of the fitted sinusoid from the measured response curve.

Data analysis

Response amplitudes obtained from all animals participating in a given type of experiment were averaged. The standard error of the mean was calculated for each average and corrected for the bias resulting from small sample sizes (Sokal and Rohlf 1981). The performance of the animals under different experimental conditions were compared on the basis of the averaged response amplitudes by means of the $t$-test of the difference between two means (Rohlf and Sokal 1981; Sokal and Rohlf 1981). Because animals were not marked individually, it cannot be excluded that the same animal participated more than once in an experiment.

\section{Results}

General properties of the compensatory optomotor responses

The control systems mediating compensatory optomotor responses were characterized in freely flying $M$. stellatarum, hovering in front of a dummy flower while feeding on it. The responses were elicited by vertical grating patterns moving horizontally, thus simulating rotational and translational displacements of the animal in the horizontal plane. Patterns were oscillated sinusoidally. The animals respond to translational pattern motion (TPM) by flying back and forth, thus changing their distance to the dummy periodically (Figs. 2a, 3a). While compensating for the position disturbance simulated by the translational movement of the patterns, the retinal size of the dummy flower changes: it becomes larger when the animal approaches and becomes smaller when it retreats. Such changes in apparent size are compensated for by Macroglossum, i.e. it responds to the increase or decrease of the angular size of a circular

Fig. 2a,b Five video-frames from one oscillation cycle of two different animals responding to translational (a) or rotational (b) stimulation. Pictograms on top of figure indicate direction of sinusoidal whole-screen pattern motion, displayed on two monitors at both sides of the animal (see Fig. 1): empty ( filled) arrow heads indicate front to back (back to front) motion in $a$, clockwise (counterclockwise) motion in $b$. Oscillation frequency of patterns $1 \mathrm{~Hz}$. Animals have inserted their proboscis into the dummy flower. a When responding to translational pattern motion (TPM) the animal flies back and forth, increasing and decreasing the distance to the dummy, respectively. b When responding to rotational pattern motion (RPM) the animal turns about the dummy flower. Note that the genuine Umatic tapes used for automatic frame-by-frame analysis had a better image quality than the VHS-backups the present video-frames have been taken from

dummy flower by flying back or forth, respectively (Pfaff and Varjú 1991; Farina et al. 1994). Therefore, the two compensatory responses are antagonistic in our experimental situation when employing translational stimuli (see Discussion for further comments). Note that mechanical cues derived via the proboscis, which is inserted into the flower, were demonstrated to play only a minor role in mediating translational responses (TR), at least under laboratory conditions (Farina et al. 1994). The

\section{a Translational response}
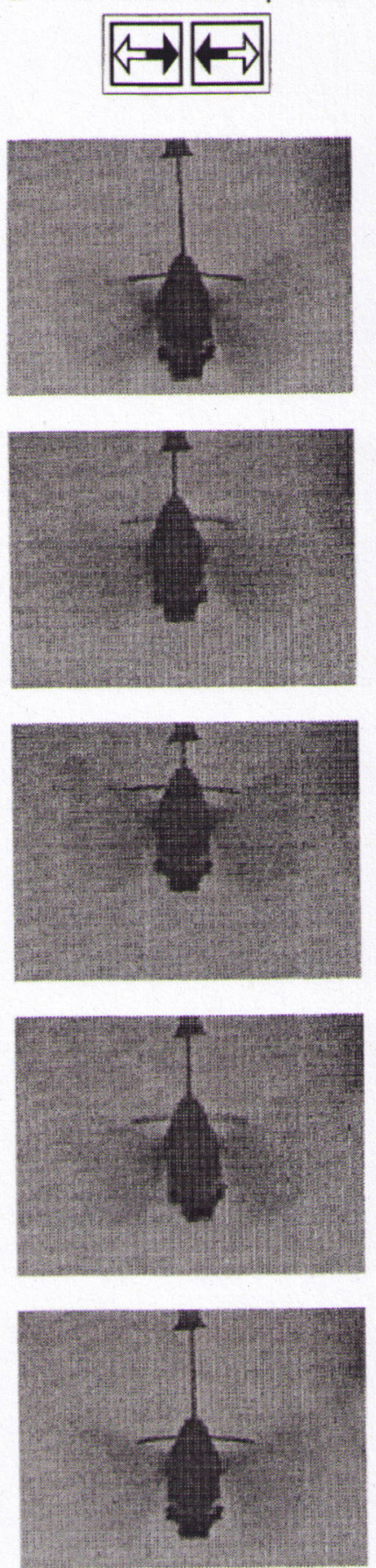

\section{b Rotational response}
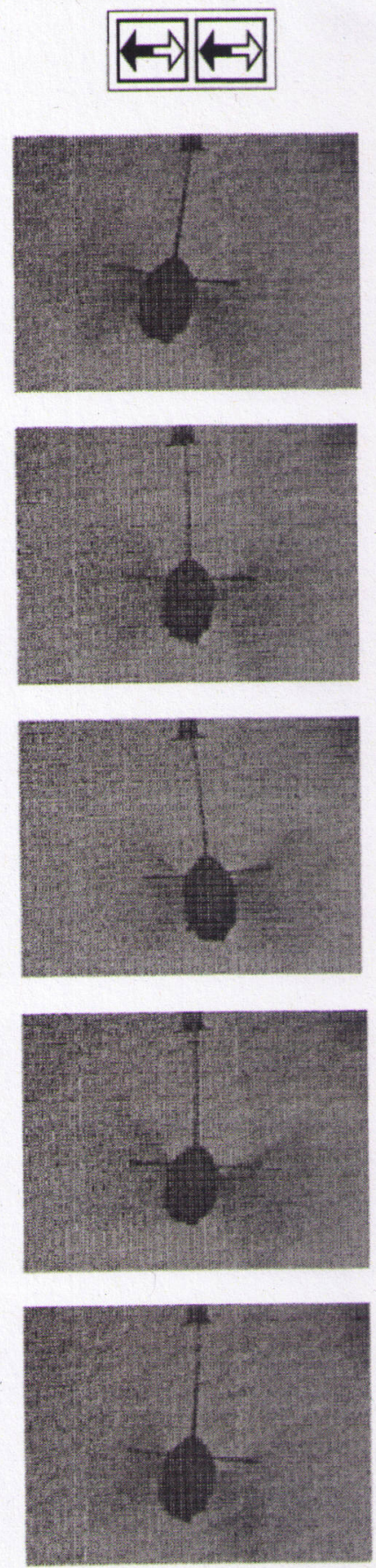


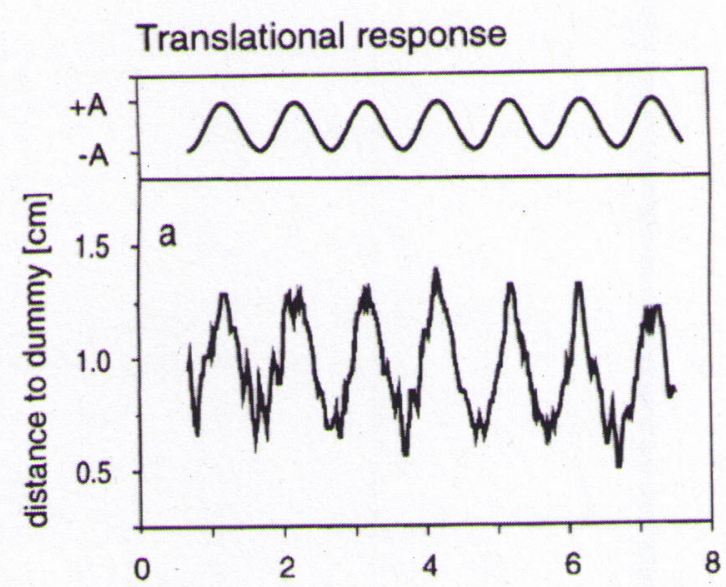

Rotational response

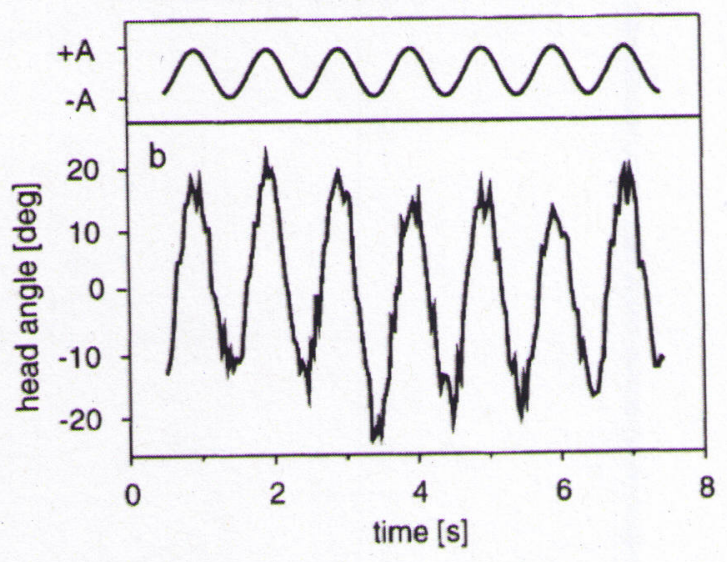

Fig. 3a, b Sample record of the response to TPM (a) and RPM (b). a Distance between the snoot of the animal and the dummy flower versus time. The animal flies back and forth; thus, the distance to the dummy flower increases and decreases, respectively. b Head angle with respect to an external coordinate system versus time. The animal turns around the dummy flower on which it is feeding. Stimulus: sinusoidal whole-screen pattern motion with frequency $=1 \mathrm{~Hz}$ and amplitude $\mathbf{A}=1 \mathrm{~cm}$ in $\mathbf{a}$ and $\mathbf{A}=23^{\circ}$ in $\mathbf{b}$

TR to patterns generated on the computer screens is basically the same as the response to mechanically moving stripe patterns as were employed by Farina et al. (1995). In the latter study the TR has been named 'drift compensating response'. If either the initial distance of the animal or the oscillation amplitude of the pattern is too large, the animal may lose proboscis contact with the dummy flower; therefore, stimulus amplitudes suitable for experimentation were limited. It is interesting to note in this context that the animals made use of the compensatory mechanisms investigated in the present study only as long as they could feed on the flower. If they had lost contact with the dummy, the animals might have even flown against the direction of TPM.

Rotational pattern motion (RPM) results in turning responses of Macroglossum (Fig. 2b). When the patterns move counterclockwise, i.e. from front to back on the left screen and back to front on the right screen (black arrows in inset of Fig. 2b), the animal flies on a circular trajectory from left to right (frames 1-3 in Fig. 2b) with the dummy flower in the centre of the circle. When the patterns move clockwise, the animal flies in the opposite direction (frames 3-5 in Fig. 2b). Obviously, the animal turns around the dummy flower rather than the centre of its thorax, i.e. it moves sideways, thereby counterrotating head and body to keep the dummy flower in the frontal visual field and maintaining the distance to the dummy flower. This type of turning response indicates again that the flower is of major importance for the position-stabilizing responses. When analysing the rotational responses (RR), the change of head angle with respect to an external coordinate system has been determined over time (e.g. Fig. 3b).

Dynamic properties of the compensatory optomotor responses

In order to investigate the dynamic properties of the optomotor system, compensatory responses were elicited by translational or rotational motion of whole-screen patterns for oscillation frequencies ranging from $0.1 \mathrm{~Hz}$ to $8 \mathrm{~Hz}$. The response to TPM (Fig. 4a) remains about the same for frequencies between $0.1 \mathrm{~Hz}$ and $0.75 \mathrm{~Hz}$ but then increases and reaches a peak between $2 \mathrm{~Hz}$ and $3 \mathrm{~Hz}$. Towards higher frequencies the response decreases steeply. The frequency dependence of the response to RPM (Fig. 4c) differs only slightly from that to TPM. The response amplitudes decrease somewhat in the range $0.1-1 \mathrm{~Hz}$ but increase steeply towards higher oscillation frequencies. At $4 \mathrm{~Hz}$ they reach a narrow peak and decrease steeply towards higher frequencies.

The overall similarity of the dynamic properties of translational and rotational responses is further reflected in the very similar frequency dependence of the phase shift (Figs. 4b,d). The phase shift is about zero up to frequencies of about $1 \mathrm{~Hz}$; at higher frequencies the response lags more and more behind the stimulus.

\section{Unilateral versus bilateral stimulation}

Responses to unilateral and bilateral pattern motion were compared in order to elucidate whether specific interactions between the motion signals from both eyes are involved in stabilizing the position of the animal. Unilateral motion induces both a TR and a RR component (Fig. 5): the animals fly back and forth. Simultaneously they turn clockwise during forward flight and counterclockwise during backward flight when, for example, the pattern is displayed on their left side. The TR and RR components to unilateral motion were significantly smaller than the TR and RR to the corresponding bilateral stimuli (Fig. 5, a $<0.001$ ). For the TR component this is in accordance with an earlier study on Macroglossum (Farina et al. 1995). In order to characterize potential interactions of motion signals from both eyes, a prediction of the response to bilateral motion 

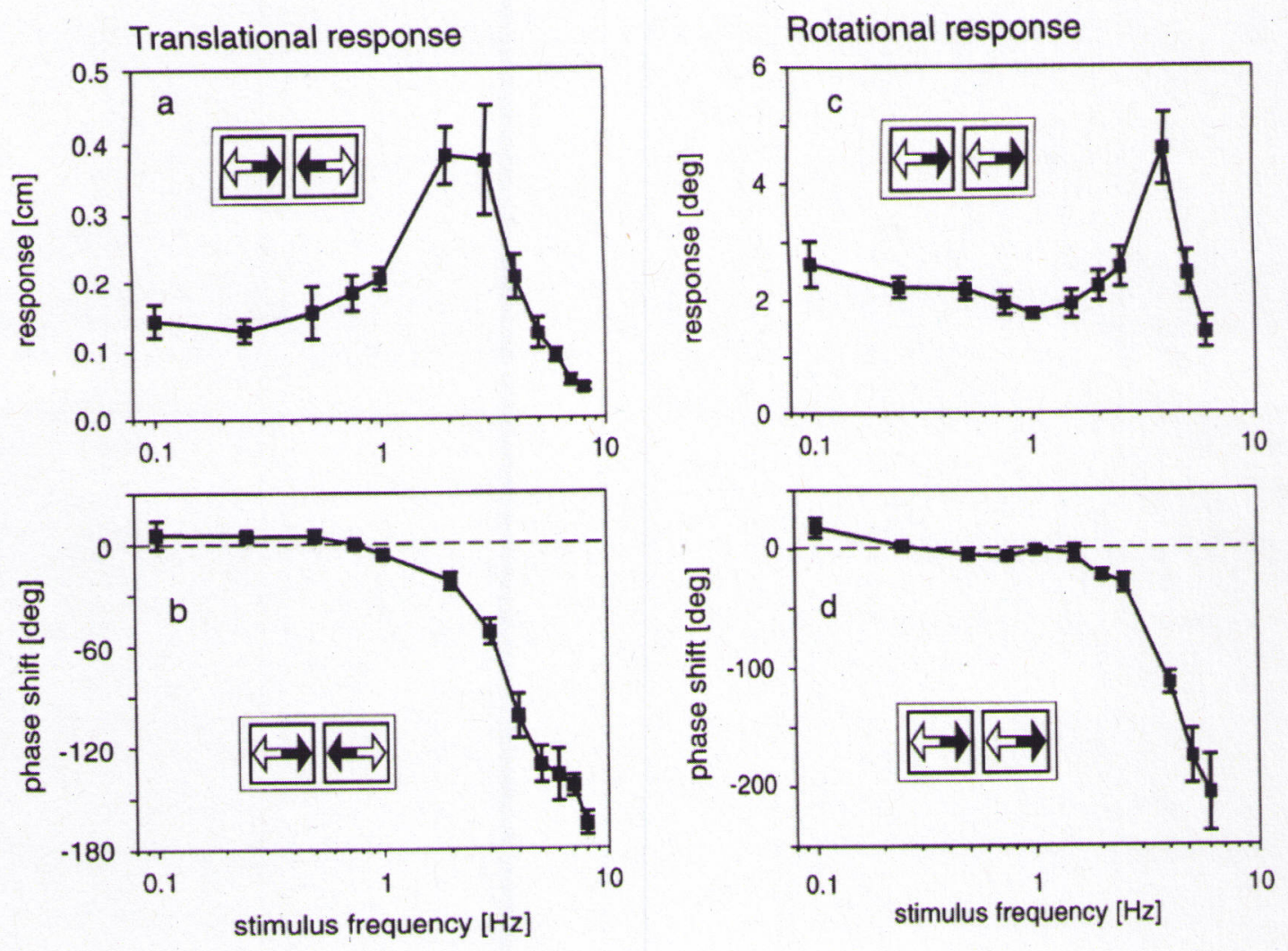

Fig. 4a-d Average amplitudes and phase shifts \pm SEM of the responses to TPM $(\mathbf{a}, \mathbf{b})$ and RPM $(\mathbf{c}, \mathbf{d})$ as a function of oscillation frequency of the stimulus pattern. Experimental data were derived by evaluating the mean amplitudes of at least six cycles of sample records at oscillation frequencies of $0.5 \mathrm{~Hz}$ and higher, and three cycles at oscillation frequencies below $0.5 \mathrm{~Hz}$. The data points are based on measurements obtained with 5-26 animals (TPM) and 4-22 animals (RPM), respectively. Oscillation amplitudes: translation $0.5 \mathrm{~cm}$, rotation $4.6^{\circ}$. The dynamic properties of the translational and rotational responses are very similar with respect to both the response amplitude and the phase shift

based on the respective response components to unilateral motion was calculated and compared to the corresponding measured response (Fig. 5). The prediction was calculated by linear superposition of the responses induced by unilateral motion on either side of the animal, assuming no specific interactions between the motion signals from both eyes. The predicted response amplitudes are about the same as the measured ones, the small difference is not significant. This holds for both the responses to TPM (Fig. 5a, a $>0.4$ ) and RPM (Fig. 5b, a $>0.5$ ). Patterns were oscillated with a frequency of $1 \mathrm{~Hz}$ and an amplitude of $1 \mathrm{~cm}$ or $9.2^{\circ}$, respectively.

Superposition of translational and rotational stimulus components

So far, bilateral motion stimuli were employed to simulate either translational or rotational position changes of the animal. Since under natural conditions transla-

tional and rotational position changes are usually superimposed, we analysed to what extent, if at all, both components are compensated for during combined RPM and TPM. To obtain combined TPM and RPM, different oscillation amplitudes were chosen for the left and the right pattern: left pattern $0.5 \mathrm{~cm}\left(4.6^{\circ}\right)$ and right pattern $1 \mathrm{~cm}\left(9.2^{\circ}\right)$, or vice versa.

Under this stimulus regime the animals fly back and forth along a curved path. Hence, both the distance of the animal to the dummy flower as well as the head angle change. As a consequence, the retinal image slip resulting from both the translational and the rotational stimulus component is reduced by the response of the animal. The TR component (Fig. 6a) is three to four times larger when the main stimulus component is translational rather than rotational, i.e. when the patterns on both sides of the animal move from front to back or from back to front though with a different amplitude. On the other hand, the RR component (Fig. 6b), on average, is two to three times larger when the main stimulus component is rotational rather than translational, i.e. when the patterns on both sides move with a different amplitude, one of them moving from front to back and the other one from back to front. The TR and RR components to combined TPM and RPM are slightly smaller than, but not significantly different from, the linear sum of the TR and RR components induced by the corresponding unilateral stimuli (Fig. 7a, $a>0.1$; Fig. $7 b, a>0.2)$; thus, the two response components, TR and RR, apparently do not affect each other. 
Translational response

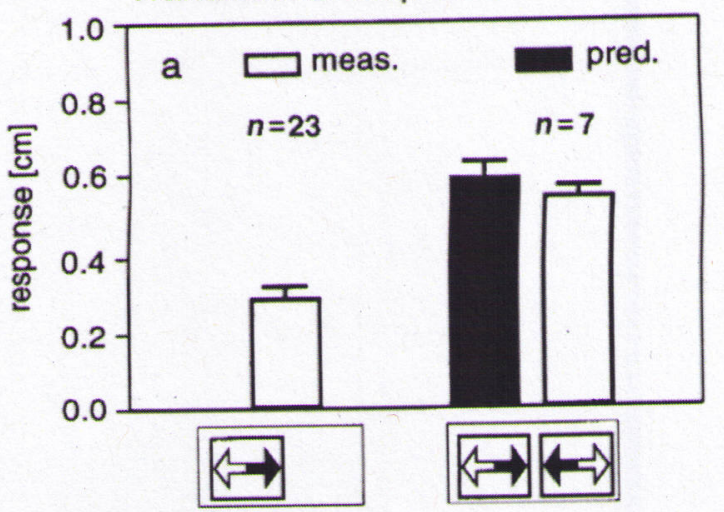

Rotational response

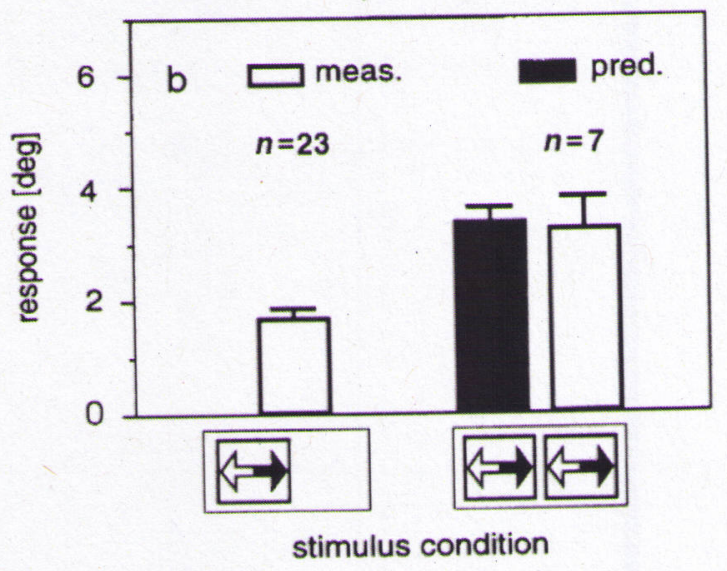

Fig. 5 a Average amplitudes (+ SEM) of the translational response component to unilateral whole-screen pattern motion presented alternately on either side and average response amplitudes (+SEM) to bilateral translational whole-screen pattern motion at an oscillation amplitude of $1 \mathrm{~cm}$. Frequency $1 \mathrm{~Hz} . n=$ number of animals. White columns: measured responses, black columns: predicted responses. The prediction of the bilateral response is based on the linear superposition of the unilateral responses. Unilateral stimulation is indicated by pictograms with an arrow only in the left box though unilateral stimuli were presented alternately on the left and the right screen. The responses to unilateral motion with the pattern on the left or the right screen were pooled. b Average amplitudes (+SEM) of the rotational response component to unilateral whole-screen pattern motion on either side and average response amplitudes (+ SEM) to bilateral rotational whole-screen pattern motion at an oscillation amplitude of $9.2^{\circ}$. For other conventions see a. The measured responses to TPM and RPM are virtually as large as predicted

\section{Functional regionalization of the eye}

TPM in the upper or lower halves of the screens leads to approximately the same response amplitude (Fig. 8a, $a>0.1$, suggesting that the sensitivity of the system controlling translation is independent of the vertical position of the motion stimulus. The response to motion in either half of the screens amounts to approximately two-thirds of the response to simultaneous motion in both halves. Hence, the response to whole-screen stimulation is smaller than would be expected from linear
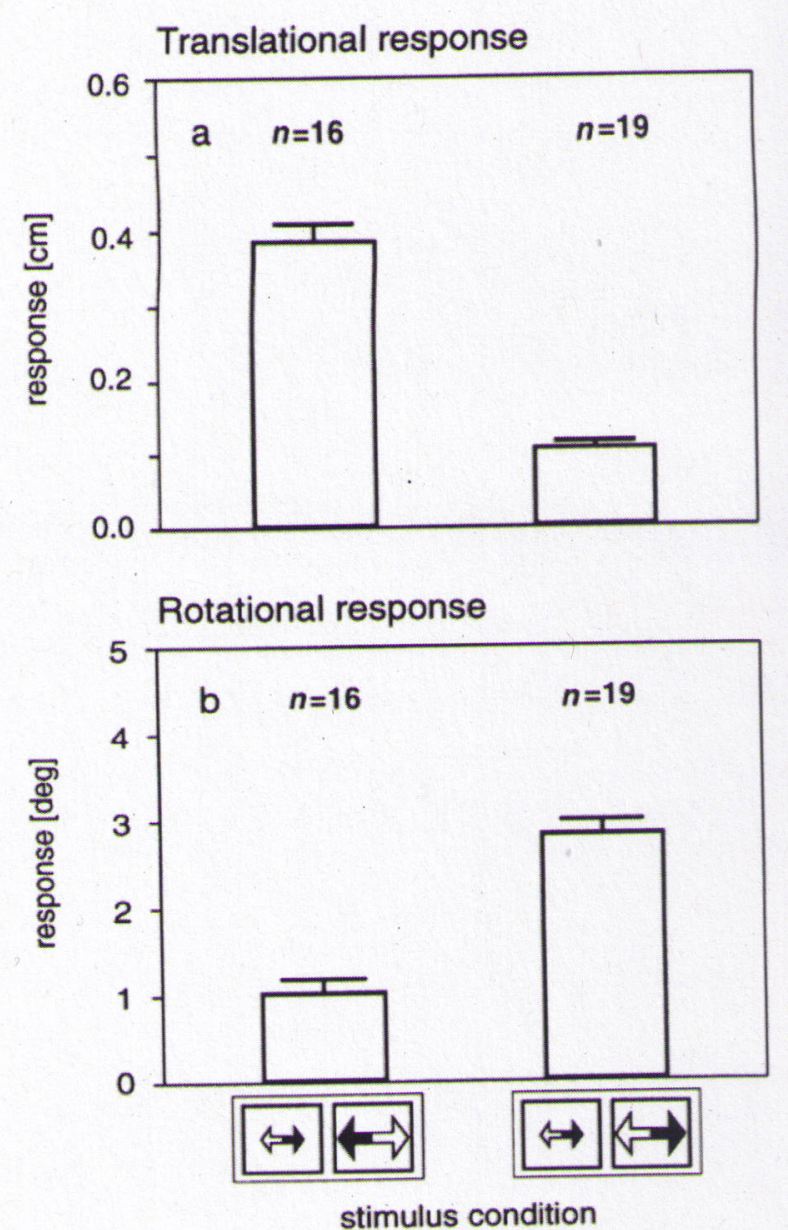

Fig. 6a, b Average amplitudes (+SEM) of translational response (TR) (a) and rotational response (RR) (b) components to superimposed TPM and RPM. Stimulus parameters: oscillation amplitude indicated by the pictograms (small arrows: $0.5 \mathrm{~cm}, 4.6^{\circ}$; large arrows: $\left.1 \mathrm{~cm}, 9.2^{\circ}\right)$; oscillation frequency $1 \mathrm{~Hz} . n=$ number of animals. The main stimulus component is either translational (left pictogram) or rotational (right pictogram). a The TR component is larger when the main stimulus component is translational rather than rotational. b The opposite is true for the RR component

summation of the responses to motion in each part alone (Fig. 8a, a $<0.01$ ).

In contrast to the response to TPM, the response to RPM strongly depends on the vertical position of the stimulus: the RR to RPM in the upper part of the visual field is much larger than the RR to RPM in the lower part (Fig. $8 b, a<0.001$ ). The measured response to whole-screen stimulation is somewhat smaller than the response predicted on the basis of the linear summation of the responses to motion in each part of the visual field alone but the difference is not significant (Fig. 8b, a $>0.1$ ). Similar results are obtained for oscillation amplitudes of $9.2^{\circ}$ (shown in Fig. 8b) and $23^{\circ}$ (not shown; Kern 1994).

TPM in the fronto-lateral parts of the visual field results in responses significantly stronger than those to 
Translational response

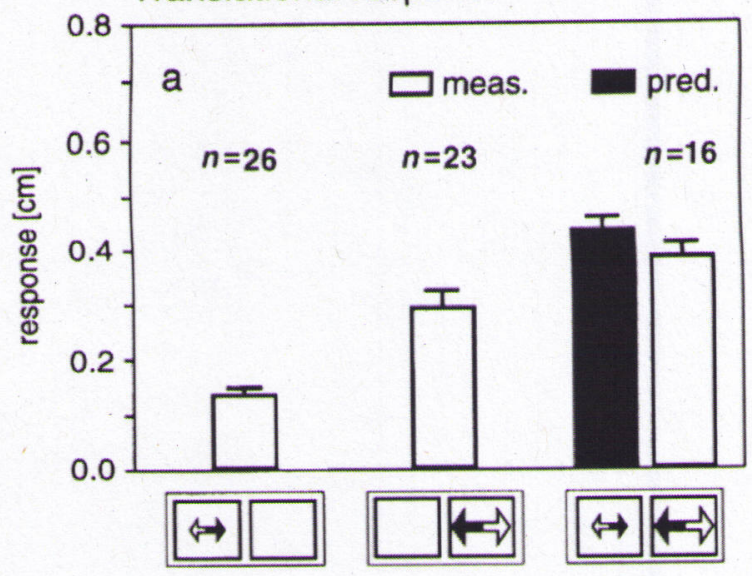

Rotational response

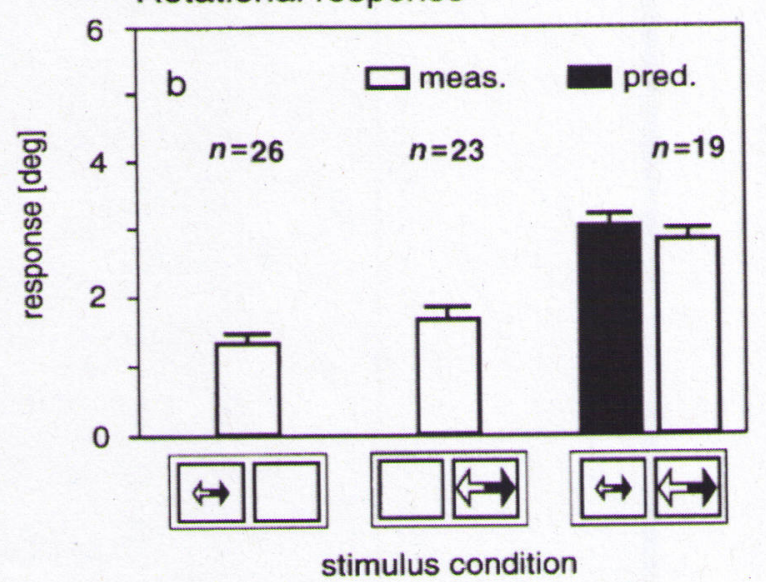

Fig. 7a, b Average amplitudes (+ SEM) of the TR (a) and RR (b) components to superimposed TPM and RPM compared to the prediction from the linear superposition of the responses to the corresponding unilateral stimuli. Unilateral stimulation is indicated by pictograms with an arrow only in the left or right box though unilateral stimuli were presented alternately on the left and on the right screen. The responses to unilateral motion with the pattern on the left or on the right screen were pooled. Stimulus parameters: oscillation amplitudes indicated by the pictograms (small arrows: $0.5 \mathrm{~cm}, 4.6^{\circ}$; large arrows: $1 \mathrm{~cm}, 9.2^{\circ}$ ), oscillation frequency $1 \mathrm{~Hz}$. During bilateral stimulation the oscillation amplitude was small (large) on the left (right) screen or vice versa (only indicated by the pictograms for one of the stimulus situations). The data for both stimulus situations were pooled. $n=$ number of animals. White columns: measured responses (meas.), black columns: predicted responses (pred.). Stimulus conditions: bilateral stimulus is mainly translational (a) or rotational (b). Both the translational (a) and the rotational (b) response component are almost as large as predicted on the assumption of linear superposition

TPM in the lateral parts (Fig. 9a, a $<0.01$ ). Likewise, the TR component to unilateral pattern movement in the fronto-lateral part of the visual field is significantly larger than to unilateral movement in the lateral part $(a<0.001$; not shown in Fig. 9a). Bilateral pattern motion in both the fronto-lateral and the lateral areas of the visual field is more effective than the corresponding unilateral stimulation. The measured responses are ei-

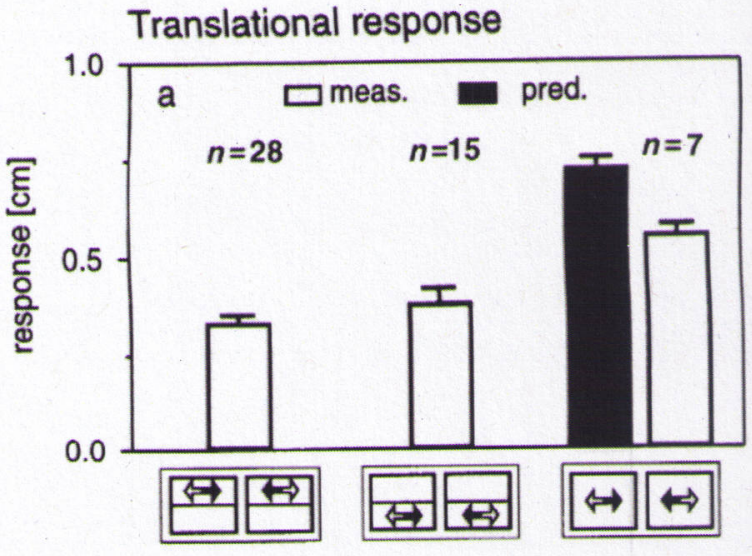

Rotational response

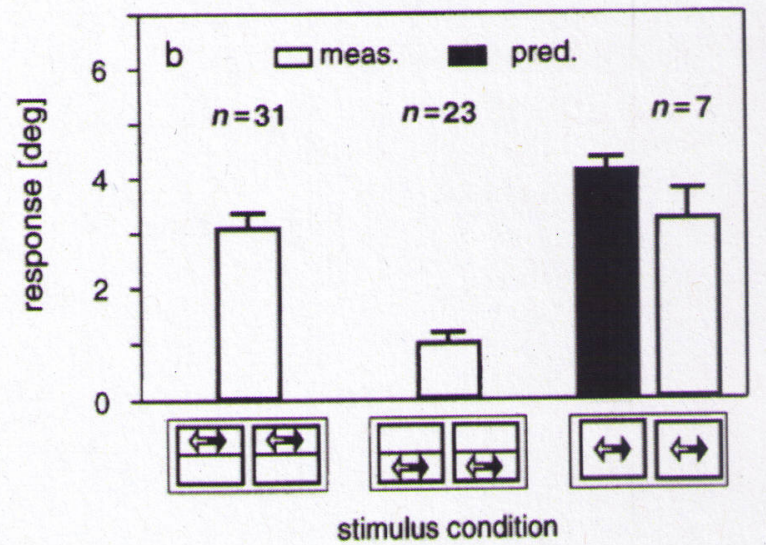

Fig. 8a, b Average response amplitudes (+ SEM) to translational (a) and rotational (b) stimuli in the upper or lower halves of the screens only, or within the whole screen. Stimulus conditions: oscillation amplitude $1 \mathrm{~cm}\left(9.2^{\circ}\right)$; oscillation frequency $1 \mathrm{~Hz} . n=$ number of animals. White columns: measured responses (meas.), black columns: predicted responses (pred.). The prediction of the response to wholescreen motion is based on the linear superposition of the responses to the partial stimuli. a The translational system is about equally sensitive to motion in both dorsal and ventral areas of the visual field. b The rotational system is much more sensitive to dorsal than to ventral stimulation

ther about as large as predicted (Fig. 9a, right hand side, $a>0.05$ ) or considerably smaller (Fig. 9a, left hand side, $a<0.001)$.

In contrast to the response to TPM, the response to RPM is significantly larger when the patterns move in the lateral parts of the visual field than when they move in the fronto-lateral parts (Fig. 9b, a < 0.001). A similar response is seen for the RR component to unilateral pattern motion ( $a<0.001$; not shown in Fig. 9b). Bilateral stimulation is more effective than unilateral stimulation. Irrespective of the pattern location within the visual field, the RRs to bilateral pattern motion are about the same as the responses predicted by assuming linear summation of the responses induced by the corresponding unilateral motion stimuli (Fig. 9b, $a>0.1)$. 
Translational response

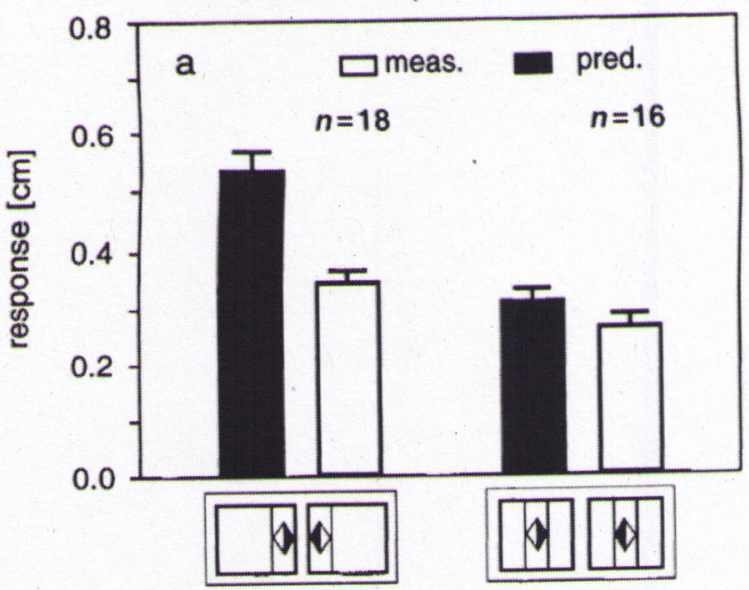

Rotational response

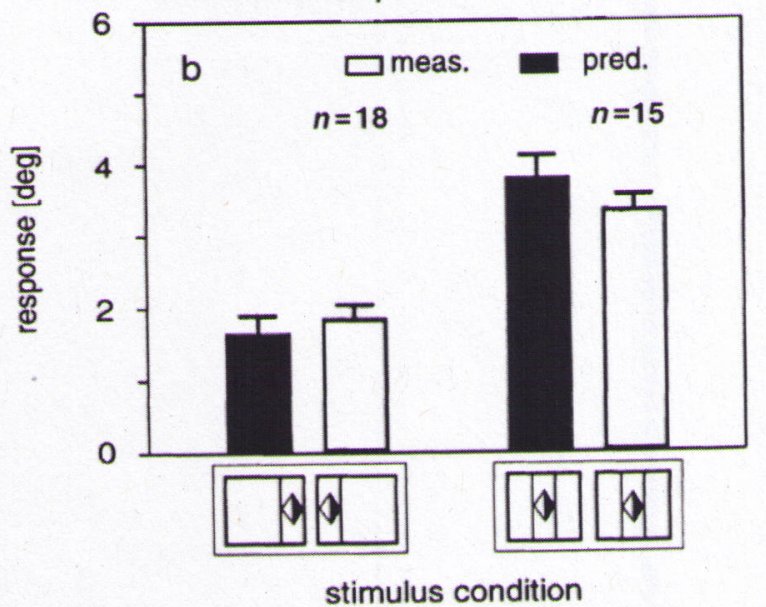

Fig. 9a, b Average response amplitudes (+SEM) to translational (a) and rotational (b) stimulation in limited areas of the screens compared to the linear sum of the average responses to the corresponding unilateral motion stimuli (not shown). Stimulus conditions: oscillation amplitudes in anterior areas of the screens: $0.7 \mathrm{~cm}\left(6^{\circ}\right)$, in medial areas of the screens: $0.9 \mathrm{~cm}\left(5 \cdot 5^{\circ}\right) \cdot n=$ number of animals. White columns: measured responses (meas.), black columns: predicted responses (pred.). Note that the responses to unilateral motion were obtained with the pattern on the left or on the right screen. a The translational response is larger when the patterns move in fronto-lateral rather than in lateral areas of the visual field. b The opposite holds for the rotational response

Dependence of the response on distance between animal and dummy flower

It is a striking feature of the TR that it depends on the distance of the animal to the dummy flower: the shorter the distance the weaker the response, as shown in Fig. 10a for a specific stimulus condition (see also Farina et al. 1995). Therefore, one might argue that differences between the average TR obtained under different stimulus conditions result from differences between the distances to the dummy flower of the animals participating in the respective experiments. To test for
Translational response

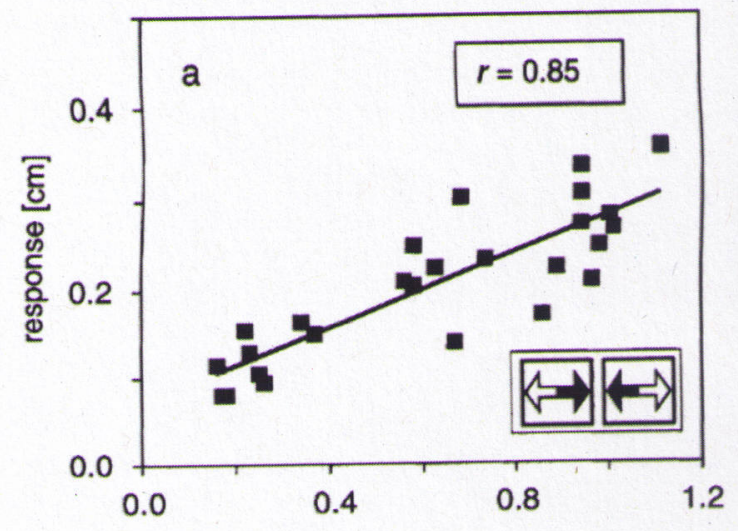

Rotational response

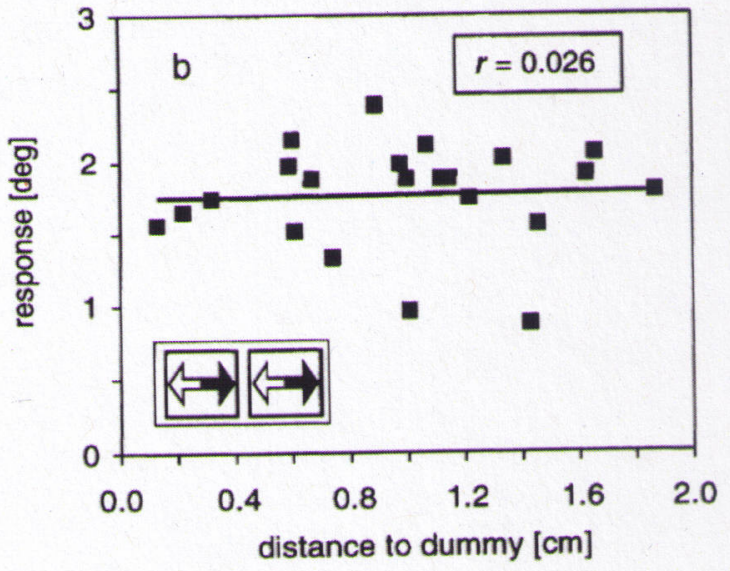

Fig. 10a,b Amplitude of TR (a) and RR (b) versus distance of the animals to the dummy. Stimulus parameters: sinusoidal whole-screen pattern motion, oscillation frequency $1 \mathrm{~Hz}$; amplitude $0.5 \mathrm{~cm}\left(4.6^{\circ}\right)$. Each data point represents the response amplitude of a single animal and its mean distance to the dummy flower. The distance was averaged over all oscillation cycles evaluated for the TR and RR, respectively. a The TR amplitude increases linearly with the mean distance (correlation coefficient $r=0.85$, number of animals: 26). b No such correlation can be found for the RR (correlation coefficient $r=0.026$, number of animals: 22 )

this possibility, the average distance of the animals participating in a given experiment was evaluated. It was examined by means of the $t$-test whether the average distances obtained for experiments that in the previous sections were compared to each other, differed significantly. Only in a single case was a significant difference detected: the average distance of the animals stimulated by unilateral motion of a pattern of two periods displayed in the anterior region of a screen was significantly larger than the average distance of the animals stimulated by motion of the same pattern presented bilaterally $(a<0.05)$. The predicted response in this case is much larger than the measured one (Fig. 9a, left side). Therefore, the question arises whether this relationship between measured and predicted response has been affected qualitatively by the differences in distance of the 
animals participating in the respective experiments. We doubt this possibility for two reasons. Firstly, throughout the experiments the measured responses were not significantly larger than the predicted ones. Secondly, the dependence of the TR on distance, as read from the slope of the regression line through the data points for the respective experiments (not shown), does not account for such a dramatic change in response strength as would be necessary to make the measured response larger than the predicted one.

In contrast to the response to TPM, the RR does not depend consistently, for all stimulus conditions, on the distance between the animal and the dummy flower. The slopes of the regression lines through the data points obtained in experiments with rotational stimulation as well as the corresponding correlation coefficient are rather small (c.f. Fig. 10b).

\section{Discussion}

Both translational and rotational compensatory optomotor responses of the diurnal hummingbird hawk moth, M. stellatarum, were characterized. The experiments were performed with unrestrained animals which hovered in front of a dummy flower while feeding on it. The animals fly back and forth during TPM or turn about the dummy flower during RPM. The response to unilateral stimulation consists of both a translational and a rotational component as does the response to those bilateral motion stimuli that contain superimposed translational and rotational components. The TR and the RR component do not seem to influence each other. The sensitivity of the optomotor system to TPM and RPM varies within the visual field. Whereas the translational system is most sensitive to pattern motion in the fronto-lateral parts of the visual field along its entire vertical extent, the rotational system responds best to motion in the lateral and dorsal parts. The responses to composite stimuli are smaller than, or virtually the same as, those predicted by linear summation of the responses to the corresponding partial stimuli alone.

In contrast to the present study, no RR component could be detected in the responses of Macroglossum to unilateral pattern movement in a previous analysis (Farina et al. 1995). This difference might be due to the fact that different eye regions were stimulated in the present and the previous study. Moreover, in the previous study the rear borders of the stimulus setup might have reduced the responses. Such borders were occluded in the present study (see Materials and methods).

Our finding that compensatory TRs and RRs during position stabilization seem to be controlled independently of each other in Macroglossum suggests that the compensation of rotational deviations from straight flight is not compromised by the simultaneously occurring translational component. Such an independence has been shown to be present in locusts (Baader et al. 1992; see, however, Preiss and Spork 1995). Also, in humans, independent mechanisms for the detection of expansion and rotation in retinal image flow have been suggested on the basis of psychophysical experiments (Regan and Beverley 1985; Freeman and Harris 1992).

\section{Dynamics of the systems}

The frequency characteristics of the responses to both TPM and RPM resemble the response of a band-pass filter. Both the frequency and the phase-shift characteristics of the TR are very similar to those determined in a previous study on Macroglossum (Farina et al. 1995). The dynamic properties of the rotational control system in Macroglossum differ from those in the hoverfly (Collett 1980b) and in the housefly (Egelhaaf 1987). In both fly species the frequency characteristic resembles that of a low-pass filter with a cut-off frequency of about $0.1 \mathrm{~Hz}$. Since flowers shaken by wind may oscillate at frequencies much higher than $0.1 \mathrm{~Hz}$ (Farina et al. 1994), the higher-frequency cut-off of the optomotor system of Macroglossum may well be a specific adaptation to the demands of its feeding behaviour. The low cut-off frequency of the optomotor response in both fly species was discussed as an adaptation to avoid interference with a second parallel motion sensitive system, a tracking and fixation system, which is more sensitive to higher oscillation frequencies (Collett 1980b; Egelhaaf 1987).

\section{Intraocular and interocular interactions}

Motion in extended areas of the visual field contributes to the control of translation and rotation in Macroglossum. Hence, local motion signals from different parts of each eye as well as from both eyes have to be pooled somewhere before the motor output. Motion in front of both eyes leads to responses that are smaller than or approximately as large as those predicted by linear summation of the responses to the corresponding partial stimuli. A similar relation holds when the responses to bilateral motion in parts of the screens are compared to the responses to whole-screen stimulation. These findings are in line with the notion that the motion-induced retinal signals are added and then underly saturation non-linearities. However, our results cannot be explained completely by such a simple scheme, since deviations of the response to combined stimuli from the linear prediction are not correlated with the strength of the responses. The reasons for this discrepancy are not yet known but might simply be related to the fact that the experiments were performed with unrestrained animals. For example, during unilateral pattern presentation the animals shift the orientation of their whole body by $10-20^{\circ}$ towards the side with the pattern. In contrast, during bilateral pattern presentation, on average, the animal is aligned with the bisector of the angle between the two screens. Consequently, slightly different eye re- 
gions were stimulated during unilateral, respectively, bilateral pattern motion. In combination with the functional regionalization of the eyes these variations may partly account for the discrepancy. Similar kinds of interactions according to a summation-saturation scheme are found in other insects (c.f. fly: Götz 1968; Reichardt et al. 1983; gypsy moth: Preiss 1991; bee: Moore et al. 1981; Moore and Rankin 1982).

In the optomotor systems of other species more sophisticated interactions between signals originating from different eye regions than those found in Macroglossum have been described. In crabs (Nalbach et al. 1989; Kern et al. 1993), waterstriders (H. Dahmen, personal communication) and pigeons (Nalbach 1992) it was demonstrated that the response to rotational motion of two patterns presented at widely separated locations in the visual field is significantly stronger than predicted from the linear sum of the responses to motion of each of these patterns alone. The enhancement is most pronounced if the patterns are separated by $180^{\circ}$ (e.g. Nalbach et al. 1989) and has been discussed as a mechanism leading to separate representations of translational and rotational self-motion. In Macroglossum, no significant difference has been found between predicted and measured responses to widely separated stimuli (Fig. 9b); thus, it does not take advantage of increased angular stimulus separation.

\section{Drift compensation and distance stabilization}

TRs of Macroglossum are not only induced by stripe patterns moving in the fronto-lateral part of the visual field (present study; Farina et al. 1995), but also when the angular size of a circular dummy flower, seen in the frontal and fronto-lateral visual field, increases or decreases (Pfaff and Varjú 1991; Farina et al. 1994). Hence, the question arises whether the TRs measured under the two stimulus conditions are mediated by the same system. In an earlier study (Farina et al. 1995) it was suggested that they are mediated by two separate systems: a drift-compensating and a distance-stabilizing system. This suggestion was mainly based on differences in the dynamic properties of the responses. In natural situations, each of the two proposed systems promotes compensatory responses aimed at keeping the animal in contact with the flower. Only under the artificial conditions of our and the former (Farina et al. 1995) stimulus paradigms are the distance-stabilizing and the driftcompensating responses antagonistic. On the basis of the responses to the stimuli used in the present study it is not possible to decide whether one or two systems are involved in controlling the distance to flowers. In any case, information from fronto-lateral parts of the visual field of the animal is most important for the translational compensatory responses.

In the present experiments it was observed that the animals flew against the direction of TPM if they had lost proboscis contact with the flower. This observation indicates that the gain of the optomotor system of the animal is context dependent. A similar conclusion has been drawn for the translational optomotor system of freely flying guard bees Trigona (Tetragonisca) angustula. These bees respond to optomotor stimuli only as long as they can keep the nest entrance in frontal areas of the visual field (Kelber and Zeil 1990). Moreover, the optomotor system of tethered flying gypsy moths ( $L y$ mantria dispar L.) effectively controls flight altitude and speed only in the presence of the male-attracting sexpheromone (Preiss and Kramer 1983).

Functional regionalization of the eye - adapting the optomotor system to a specific task?

The retinal image displacements during rotation of the animal do not depend on the distance between object and animal. In contrast, retinal image displacements owing to translation depend on the distance of the objects from the eye: the smaller the distance the larger the retinal image displacements. Therefore, the detection of distance changes by the system responding to TPM is less impaired by simultaneous rotations when the object is close by rather than far away. On the other hand, the detection of rotation is less impaired by simultaneous translations when the objects are far away rather than close by. We suggest that Macroglossum might take advantage of these relations when compensating for position disturbances during feeding on flowers. While feeding, the flower or other parts of the plant are nearby (length of proboscis maximal $3 \mathrm{~cm}$ ) and cover frontal and fronto-lateral areas of the dorsal and ventral visual field. Hence, the increased sensitivity of the translational optomotor system of Macroglossum in fronto-lateral areas of the visual field and its similar sensitivity in dorsal and ventral parts can be interpreted to account for the relations just described. On the other hand, the increased sensitivity of the rotational system in dorsolateral parts of the visual field seems to be well matched to the visual environment likely to be encountered during feeding, where objects in these areas are usually more distant than in the frontal and ventral parts of the visual field.

In conclusion, the sensitivity gradients of the translational and the rotational system along the horizontal and vertical extent of the visual field suggest a functional regionalization of the eye with respect to the analysis of retinal image flow as experienced when hovering in front of a flower.

An effective control of flight by the optomotor system is not only required while hovering in front of flowers. As in other flying animals, Macroglossum needs to control its flight direction, speed over ground and altitude when cruising around. It can be expected that the optomotor system is then working under different constraints since the visual surround of the animal looks different from that in the feeding situation. Consequently, to control, for instance, its translation in be- 
havioural contexts other than feeding on flowers, Macroglossum might exploit motion information from other parts of the visual field. In the gypsy moth, for example, the control of translational disturbances is restricted to motion in the ventral parts of the visual field (Preiss 1991). Similarly, Drosophila shows compensatory ground-speed controlling responses to horizontal motion of a floor pattern beneath it (David 1979a,b).

A higher sensitivity in dorsal than in ventral areas of the visual field as found for the rotational system of Macroglossum has been described for some crab species (Kunze 1963, 1964; Korte 1968; Nalbach and Nalbach 1987). The sensitivity distribution has been interpreted as an adaptation to facilitate the extraction of the rotational component of the retinal flow in the flat habitats of these crabs (Nalbach and Nalbach 1987). Waterstriders, living on flat water surfaces, are able to separate translational and rotational self-motion even if contours are only present in small distinct areas of the visual field above the horizon. They seem to possess visual mechanisms which make use of the fact that in some areas of the visual field the interference between translational and rotational components of the optic flow is weaker than in others (Junger and Dahmen 1991). Also Macroglossum seems to take advantage of the fact that its visual environment is to some extent predictable in certain vital behavioural contexts. This may allow for a reduction in the computational expenditure needed to cope with the demands of specific behavioural tasks.

Acknowledgements The authors are particularly grateful to $\mathrm{H}$. Dahmen and M. Egelhaaf for valuable discussions throughout the experiments. M. Egelhaaf, B. Kimmerle and A.-K. Warzecha deserve thanks for critically reading the manuscript. We are indebted to $\mathrm{H}$. Bendele for expert computer and video support, and to U. Hénique, L. Forzin and W. Pix for maintaining the experimental animals. M. Pfaff was a rich source of insight into the biology of Macroglossum. We thank H. Dahmen for his least-square algorithm and J. Eikermann for drawing part of the figures. The experiments presented are part of the doctoral thesis of R. Kern, Faculty of Biology, University of Tübingen, supported by a BadenWürttemberg grant. The study was supported also by a DFG-grant to D. Varjú (SFB 307).

\section{References}

Baader A, Schäfer M, Rowell CHF (1992) The perception of the visual flow-field by flying locusts: a behavioural and neuronal analysis. J Exp Biol 165: 137-160

Blanke H (1996) Visuelle Detektion von Eigenbewegungen beim Rückenschwimmer Notonecta glauca. Doctoral dissertation, Eberhard-Karls-Universität Tübingen, Germany

Blondeau J, Heisenberg M (1982) The three-dimensional optomotor torque system of Drosophila melanogaster. J Comp Physiol A 145: 321-329

Collett TS (1980a) Some operating rules for the optomotor system of a hoverfly during voluntary flight. J Comp Physiol 138: 271282

Collett TS (1980b) Angular tracking and the optomotor response. An analysis of visual reflex interaction in a hoverfly. J Comp Physiol 140: 145-158

Collett TS, Land MF (1975) Visual control of flight behaviour in the hoverfly Syritta pipiens L. J Comp Physiol 99: 1-66
Collett TS, Nalbach H-O, Wagner H (1993) Visual stabilization in arthropods. In: Miles FA, Wallman J (eds) Visual motion and its role in the stabilization of gaze. Elsevier, Amsterdam, pp 239-263

Dahmen H, Wüst R, Zeil J (1997) Extracting egomotion parameters from optic flow: principal limits for animals and machines. In: Srinivasan MV, Venkatesh S (eds) From living eyes to seeing machines. Oxford University Press, Oxford, pp 174-198

David CT (1979a) Height control by freely-flying Drosophila. Physiol Entomol 4: 209-216

David CT (1979b) Optomotor control of speed and height by freeflying Drosophila. J Exp Biol 82: 389-392

David CT (1986) Mechanisms of directional flight in wind. In: Payne TL (ed) Mechanisms in insect olfaction. Clarendon Press, Oxford, pp 49-57

Egelhaaf M (1987) Dynamic properties of two control systems underlying visually guided turning in house-flies. J Comp Physiol A 161: 777-783

Egelhaaf M, Borst A (1993) A look into the cockpit of the fly: visual orientation, algorithms, and identified neurons. J Neurosci 13: 4563-4574

Farina WM, Varjú D, Zhou Y (1994) The regulation of distance to dummy flowers during hovering flight in the hawk moth Macroglossum stellatarum. J Comp Physiol A 174: 239-247

Farina WM, Kramer D, Varjú D (1995) The response of the hovering hawk moth Macroglossum stellatarum to translatory pattern motion. J Comp Physiol A 176: 551-562

Freeman TCA, Harris MG (1992) Human sensitivity to expanding and rotating motion: effects of complementary masking and directional structure. Vision Res 32: 81-87

Götz KG (1968) Flight control in Drosophila by visual perception of motion. Kybernetik 4: 199-208

Heisenberg M, Wolf R (1984). Vision in Drosophila. Springer, Berlin Heidelberg New York

Hengstenberg R (1993) Multisensory control in insect oculomotor systems. In: Wallman J, Miles FA (eds) Visual motion and its role in the stabilization of gaze. Elsevier, Amsterdam, pp 285298

Junger W, Dahmen H (1991) Response to self-motion in waterstriders: visual discrimination between rotation and translation. J Comp Physiol A 169: 641-646

Kelber A, Zeil J (1990) A robust procedure for visual stabilisation of hovering flight position in guard bees of Trigona (Tetragonisca) angustula (Apidae, Meliponinae). J Comp Physiol A 167: $569-577$

Kern R (1994) Mechanisms underlying visual position stabilization in the hovering hawk moth, Macroglossum stellatarum L. Doctoral dissertation, Eberhard-Karls-Universität Tübingen, Germany

Kern R (1998) Visual position stabilization in the hummingbird hawk moth, Macroglossum stellatarum L. - II. Electrophysiological analysis of neurons sensitive to wide-field image motion. J Comp Physiol A 182: 239-249

Kern R, Nalbach H-O, Varjú D (1993) Interactions of local movement detectors enhance the detection of rotation. Optokinetic experiments with the rock crab, Pachygrapsus marmoratus. Vis Neurosci 10: 643-652

Knoll F (1922) Lichtsinn und Blumenbesuch des Falters von Macroglossum stellatarum. Abh Zool Botan Ges Wien 12: 125377

Koenderink JJ, Doorn AJ van (1976) Local structure of movement parallax of the plane. J Opt Soc Am 66: 717-723

Korte R (1968) Untersuchungen zum Sehvermögen einiger Dekapoden. Z Morp Oekol Tierehol 58: 1-37

Kunze P (1963) Der Einfluss der Grösse bewegter Felder auf den optokinetischen Augenstielnystagmus der Winkerkrabbe. Ergeb Biol 26: 55-62

Kunze P (1964) Eye-stalk reactions of the ghost crab Ocypode. In: Reiss RF (ed) Neural theory and modelling. Stanford University Press, Stanford, pp 293-305

Longuet-Higgins HC, Prazdny K (1980) The interpretation of a moving retinal image. Proc R Soc Lond B 208: 385-397 
Moore D, Rankin MA (1982) Direction-sensitive partitioning of the honeybee optomotor system. Physiol Entomol 7: 25-36

Moore D, Penikas J, Rankin MA (1981) Regional specialization for an optomotor response in the honeybee compound eye. Physiol Entomol 6: 61-69

Nalbach H-O (1992) Translational head movements of pigeons in response to a rotating pattern: characteristics and tool to analyse mechanisms underlying detection of rotational and translational optical flow. Exp Brain Res 92: 27-38

Nalbach H-O, Nalbach G (1987) Distribution of optokinetic sensitivity over the eye of crabs: its relation to habitat and possible role in flow-field analysis. J Comp Physiol A 160: 127-135

Nalbach H-O, Nalbach G, Forzin L (1989) Visual control of eyestalk orientation in crabs: vertical optokinetics, visual fixation of the horizon, and eye design. J Comp Physiol 165: 577-587

Pfaff M (1991) Visuelle Orientierung anthophiler Insekten am Beispiel des Taubenschwanzes Macroglossum stellatarum. Doctoral dissertation, Eberhard-Karls-Universität Tübingen, Germany

Pfaff M, Varjú D (1991) Mechanisms of visual distance perception in the hawk moth Macroglossum stellatarum. Zool Jahrb Physiol 95: 315-321

Preiss R (1991) Separation of translation and rotation by means of eye-region specialization in flying gypsy moths (Lepidoptera: Lymantriidae). J Insect Behav 4: 209-219

Preiss R, Kramer E (1983) Stabilization of altitude and speed in tethered flying gypsy moth males: influence of $(+)$ and $(-)-$ disparlure. Physiol Entomol 8: 55-68

Preiss R, Spork P (1995) How locusts separate pattern flow into its rotatory and translatory components (Orthoptera: Acrididae). J Insect Behav 8: 763-779
Regan D, Beverley KI (1985) Visual responses to vorticity and the neural analysis of optic flow. J Opt Soc Am A 2: 280-283

Reichardt W, Poggio T, Hausen K (1983) Figure-ground discrimination by relative movement in the visual system of the fly. Part II: towards the neural circuitry. Biol Cybern 46 [Suppl]: 130

Reichert H (1993) Sensory inputs and flight orientation in locusts. Comp Biochem Physiol 104A: 647-657

Rieger JH (1983) Information in optical flows induced by curved paths of observation. J Opt Soc Am 73: 339-344

Rohlf FJ, Sokal RR (1981) Statistical Tables. Freeman, New York

Sandeman DC (1978) Regionalization in the eye of the crab Leptograpsus variegatus: eye movements evoked by a target moving in different parts of the visual field. J Comp Physiol 123: 299-306

Sokal RR, Rohlf FJ (1981) Biometry. Freeman, New York

Spork P, Preiss R (1993) Control of flight by means of lateral visual stimuli in gregarious desert locusts, Schistocerca gregaria. Physiol Entomol 18: 195-203

Wagner H (1986) Flight performance and visual control of flight of the free-flying housefly (Musca domestica). III. Interactions between angular movement induced by wide- and smallfield stimuli. Philos Trans R Soc Lond B 312: 581-595

Wehner R (1981) Spatial vision in arthropods. In: Autrum H (ed) Handbook of sensory physiology, vol VII/6C. Comparative physiology and evolution of vision in invertebrates. Springer, Berlin Heidelberg New York, pp 287-616 